\section{Viva VENALPINA!}

Jürg Hafner (CH), Dominic Mühlberger (D) und Wolfgang Salmhofer (Lokalmatador)

Die VENALPINA IX vom 26.-31.01.2020 ist bereits Geschichte:

Wir blicken auf ein sehr hochkarätiges Programm in ausgezeichneter, freundschaftlicher Atmosphäre zurück. Am allabendlichen "Phlebo-Treff" in der Lounge des Hotels Klosterbräu inmitten von Seefeld traf sich ein Großteil der Kongressteilnehmer nach getaner Arbeit. Die KongressOrganisatoren wollten damit aus der Not eine Tugend machen, denn für einen klassischen Gesellschaftsabend fehlte schlichtweg das Geld.

Die einzelnen Kongresstage deckten jeweils ein größeres Kapitel der Phlebologie ab ganz nach dem traditionellen Format der VENALPINA. Klassische wissenschaftliche Referate wechselten mit Key Note Lectures und Mini-Reviews. Die Veranstalter möchten an dieser Stelle allen Referentinnen und Referenten sehr herzlich danken. Die Vorträge und Kurse waren allgemein hervorragend und nahmen stets auch auf die Praxis Bezug. Diese VENALPINA war für die Anwesenden ein tolles Erlebnis.

Sonntag (26.01.2020): Werner Blättler (Ehrenpräsident SGP) sprach zum hochaktuellen und hochkontroversen Thema „VeinSymptoms“ ( $\mathrm{CO}$ s). Dieses alltägliche Problem, das für uns schwer fassbar ist, hat der Autor buchstäblich jahrzehntelang mit Messungen zur Pathophysiologie, neurobiologischen Konzepten und psychiatrischen Untersuchungen mehr und mehr eingekreist - und kommt zu überraschenden und doch plausiblen Erklärungen, die letztlich auch in der menschlichen Natur wurzeln.
Die Telemedizin mit ihrem großen Potenzial und ihren hohen technischen Anforderungen an den Datenschutz waren das zweite Hauptthema dieses Nachmittags (Michael Jünger (Greifswald), Wolfgang Salmhofer (Graz)).

Montag, 27.01.2020: Jan-Dirk Studt (Zürich), Florian Prüller (Graz), Mirko Hirschl (Wien) und Paul Gressenberger (Graz) brachten die Labormedizin der Gerinnungsstörungen sowie die klassische und die moderne Antikoagulation packend und anschaulich mit der Routine sowie mit den selteneren und kritischen Situationen in Verbindung. Die direkten oralen Antikoagulanzien (DOAC/NOAC) sowie die Heparine und Heparinoide wurden mit all ihren Vor- und Nachteilen in allen gewöhnlichen und ungewöhnlichen Situationen umfassend beleuchtet.

Das zweite Hauptthema war die Kompressionstherapie in Physiologie und Pathophysiologie sowie in den relevanten klinischen Anwendungen mit großen Übersichtsreferaten und Analysen. Dazu passte der ausgezeichnete Workshop (Bernhard Partsch (Wien), Thea Westphal (Greifswald) und Christiane Stöberl (Mödling), Caroline Buset (Zürich), Michael Jünger (Greifswald)).

Dienstag, 28.01.2020: Alle verfügbaren Daten und Metaanalysen zur venösen Thromboembolie und zum postthrombotischen Syndrom wurden sehr anschaulich und kompakt zusammengefasst (Paul Gressenberger (Graz), Jan-Dirk Studt (Zürich), Manfred Cejna (Feldkirch), Michael Jünger (Greifswald), Clemens Feistritzer (Innsbruck), Gabriele Menzinger (Wien), Christiane Stöberl (Mödling)). Dazu passten die aufschlussreichen Mini-Reviews zum pelvinen Stauungssyndrom (Jürg Traber (Kreuzlingen)) und zur Pathophysiologie der Adipositas (Gabriele Faerber (Hamburg)) und deren Bezug zur chronischen venösen Insuffizienz. Die modernen Konzepte der Vaskulitiden und deren Bedeutung in der Dermatologie und Phlebologie kamen in der umfassenden Key Note Lecture zur Sprache (Bernhard Zelger (Innsbruck)). Im Workshop wurde die Wundbehandlung praxisnah geübt und diskutiert (Barbara Binder (Graz), Jürg Hafner (Zürich)).

Mittwoch, 29.01.2020: Ulcus cruris und chronische Wunden wurden unter vielen ganz neuen und modernen Aspekten abgehandelt. Das oft schwer verdauliche Thema erhielt viele diagnostische und therapeutische Glanzlichter (Eva Valesky (Frankfurt), Benedikt Weber (Wien), Stefan Salzmann (Münster), Florian Anzengruber (Zürich), Caroline Buset (Zürich), Angeliki Koulouri (Lausanne), Kornelia Böhler (Wien), Elena Conde-Montero (Madrid), Jürg Hafner (Zürich), Catherine van Montfrans (Rotterdam), Jörg Jabkowski (Linz)). Der Workshop zeigte die Praxis der arteriellen und venösen Abklärung (Karl Forstner (Salzburg), Dominic Mühlberger (Bochum), Luca Spinedi (Locarno)).

Donnerstag, 30.01.2020: Der vierte volle Kongresstag, zu welchem die chirurgisch ausgerichteten Phlebologen oft eigens anreisen, deckte die moderne Abklärung und Therapie der Varikosis in ausgezeichneten Übersichtsreferaten sehr umfassend ab (Erich Brenner (Innsbruck), Luca Spinedi (Locarno), Dominic Mühlberger (Bochum), Holger Kluess (München), Tim Wentel (Rotterdam), Manfred Baldt (Wolfsberg und Althofen), Kornelia Böhler (Wien), Sanja Schuller-Petrovic (Wien), Dominik Heim (Fruttigen/ Thun), Paolo Cassina (Lugano)). Dazu passte der Workshop mit Phantomen, welcher die praxisnahe Erprobung der modernen Varizentherapie ermöglichte (Stanislava Tzaneva (Wien), Renate Murena (Köln), Rosmarie Holzinger (Zürich), Tim Wentel (Rotterdam), Michael Kockaert (Lausanne und Zürich)). Die Key Note Lecture fokussierte auf die 


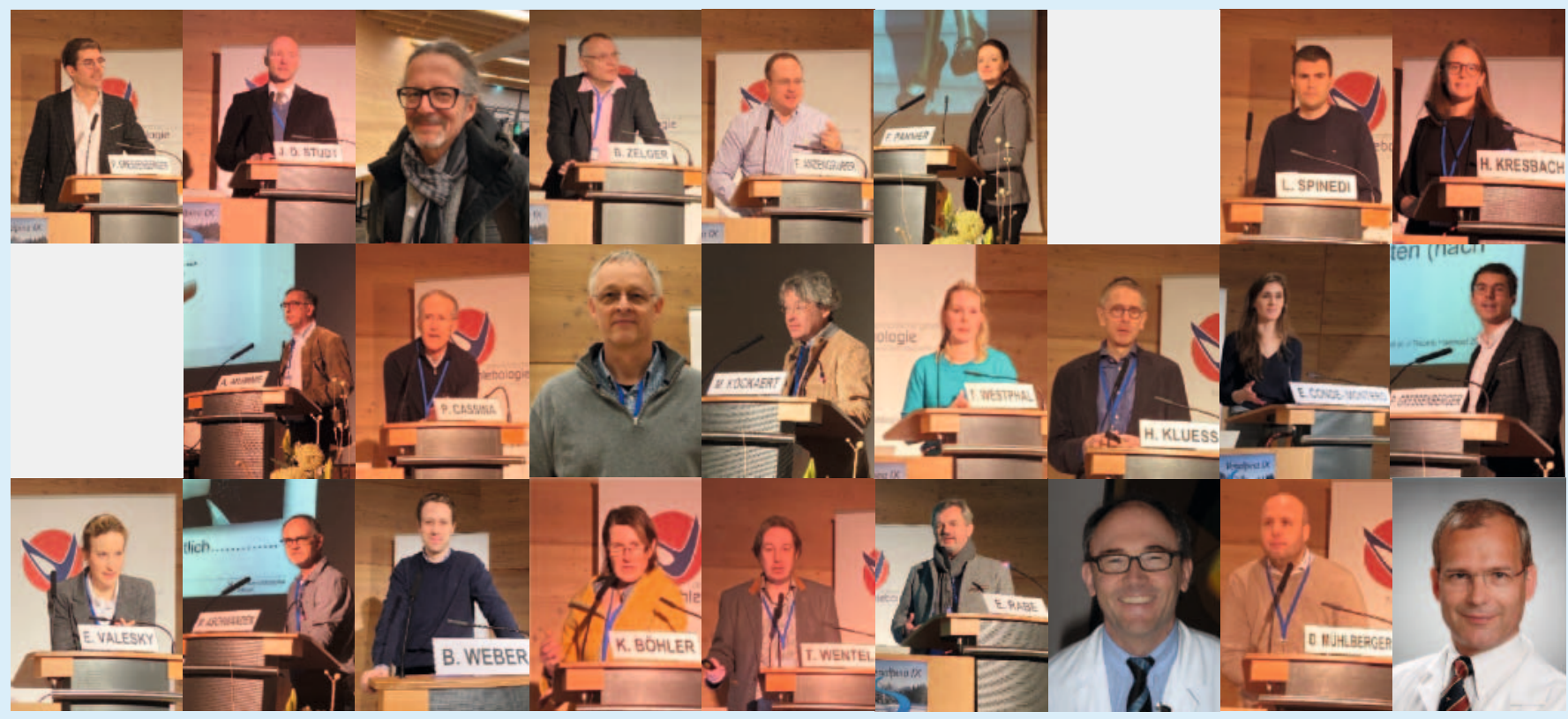

Referenten der Venalpina IX.

Klinik der Lymphologie sowie den Stellenwert der rekonstruktiven Lymphchirurgie (Stefan Wagner (Bad Zurzach)).

Freitag, 31.01.2020: Zum Abschluss beleuchtete der Freitagvormittag einige Spezialthemen: Vena saphena accessoria anterior, sekundäre (postthrombotische) Varikose, die unterschätzte Wirksamkeit und überschätzte Morbidität der Crossektomie, die elegante US-gesteuerte Schaum-Sklerotherapie sowie last not least medikolegale Aspekte in der Phlebologie (Markus Aschwanden (Basel), Michael Kockaert (Lausanne und Zürich), Achim Mumme (Bochum), Felicitas Pannier (Bonn), Dominik Mühlberger (Bochum)). Einen glänzenden Abschluss des Kongresses bildete die Key Note Lecture „What's new in Phlebology“ von Eberhard Rabe (Bonn).

Alle Anwesenden sowie die 3 Organisatoren schätzten sich überaus glücklich, dass das Abenteuer VENALPINA ein weiteres Mal gewagt wurde. Der Kongress hat unsere anfänglich vorsichtigen Erwartungen bei weitem übertroffen. Es gibt wenig Vergleichbares auf dem Megamarkt der medizinischen Fortbildungen, und wir sollten in 3 Jahren auch die zehnte Austragung wieder mutig in Angriff nehmen:

VENALPINA X (22.-28.01.2023) in Pontresina

\section{IMPRESSUM}

Verantwortlich für Mitteilungen der SGP:

Prof. Dr. med. Jürg Hafner, Zürich 